\title{
Robust Stability of Markovian Jumping Genetic Regulatory Networks with Mode-Dependent Delays
}

\author{
Guang He, ${ }^{1}$ Jian-An Fang, ${ }^{2}$ and Xiaotai Wu${ }^{1}$ \\ ${ }^{1}$ Department of Mathematics, Anhui Polytechnic University, Anhui, Wuhu 241000, China \\ ${ }^{2}$ School of Information Science and Technology, Donghua University, Shanghai 201620, China \\ Correspondence should be addressed to Guang He, zwb850506@163.com
}

Received 28 August 2012; Revised 13 October 2012; Accepted 16 October 2012

Academic Editor: Gerhard-Wilhelm Weber

Copyright (C) 2012 Guang He et al. This is an open access article distributed under the Creative Commons Attribution License, which permits unrestricted use, distribution, and reproduction in any medium, provided the original work is properly cited.

The robust stability analysis problem is investigated for a class of Markovian jumping genetic regulatory networks with parameter uncertainties and mode-dependent delays, which varies randomly according to the Markov state and exists in both translation and feedback regulation processes. The purpose of the addressed stability analysis problem is to establish some easily verifiable conditions under which the Markovian jumping genetic regulatory networks with parameter uncertainties and mode-dependent delays is asymptotically stable. By utilizing a new Lyapunov functional and a lemma, we derive delay-dependent sufficient conditions ensuring the robust stability of the gene regulatory networks in the form of linear matrix inequalities. Illustrative examples are exploited to show the effectiveness of the derived linear-matrix-inequalities- (LMIS-) based stability conditions.

\section{Introduction}

In the past few years, genetic regulatory networks (GRNs) have been playing more and more important role in biological and biomedical sciences. With the study of genetic regulatory networks, scientists can gain insight into the underlying process of living systems at the molecular level; the dynamic behaviors of the GRNs in living organisms have received increasing attentions in the past decade [1-9].

Generally, GRNs can be described by two types of models, the Boolean networks models [10-12] and differential equation models [13-17]. Recently, the differential models have received an increasing amount of research attention since it can be provide detailed understanding of the nonlinear behavior exhibited by biological systems. Hence, our present 
research further examines the differential GRN models with both mode-dependent time delays and Markovian jumping parameters.

Time delays are inevitably occurred due to the slow processes of transcription, translation, and translocation or the finite switching speed of amplifiers. The theoretical models without consideration of time delays may provide wrong predictions $[15,18]$. The stability problem of genetic regulatory network with time delays has been investigated by many researches [15, 19-24]. For instance, Chen and Aihara [15] presented a different equation model for GRNs with constant time delays and proposed necessary and sufficient conditions for such GRNs. Ren and Cao [22] derived delay-dependent robust asymptotic stability criteria for a class of genetic regulatory networks with time-varying delays and parameter uncertainties. Wang et al. [24] developed a model for genetic regulatory networks with polytopic parameter uncertainties and derived delay-dependent stability criteria for such network. Moreover, due to the modeling inaccuracies and changes in the environment of the model, parameter uncertainties can be often encountered in the genetic regulatory networks. Therefore, the problem of robust stability analysis for uncertain GRNs emerges as a research topic of primary importance.

On the other hand, as shown in [25,26], GRNs with Markovian jump parameters are a system with transitions among the states governed by a Markov chain taking values in a finite set. Therefore, it is of significance to model genetic regulatory networks with hybrid system. Recently, Hybrid system with time-varying delays has received increasing attention [27, 28]. Specially, the stability of Markovian genetic regulatory networks, which are subject to mode switching (or jumping), has been thoroughly investigated in $[25,26]$. It should be pointed out that the delays in $[25,26]$ were a deterministic case. Ribeiro et al. [29] has pointed out that the transmission delay may occur randomly in GRNs and their probabilistic characteristics can often be obtained by statistical methods.

However, most of the reported works focus on the effect of a deterministic time delay case for the Markovian jumping genetic regulatory networks; a very few studies on the effect of stochastic delays have been reported.

In this paper, firstly, we deal with the stability problem of Markovian jumping genetic regulatory networks with mode-dependent delays, that is, the delay varies randomly according to the Markov state. Then, the results are extended to an uncertain case. By utilizing a new Lyapunov-Krasovskii function and a novel lemma, we derive new delay-dependent stability criteria in the form of linear matrix inequalities (LMIs), which can be easily checked by LMI Toolbox. Finally, two numerical examples are provided to show the effectiveness of the results.

Notations 1. Throughout this paper, $\mathbb{R}^{n}$ and $\mathbb{R}^{n \times m}$ denote, respectively, $n$-dimensional Euclidean space and the set of all $n \times m$ real matrices. The superscript " $T$ " denotes the matrix transposition and the notation $X \geq Y$ (resp., $X>Y$ ) where $X$ and $Y$ are symmetric matrices, which means that $X-Y$ is a positive semidefinite (resp., positive definite) matrix, $I$ is the $n \times n$ identity matrix, and $\lambda_{\max }(A)$ (resp., $\lambda_{\min }(A)$ ) represents the largest (resp., smallest) eigenvalue of matrix $A$. For symmetric block matrices or long matrix expressions, an asterisk $\star$ is used to represent a term that is induced by symmetry. Let $h>0$, and $C\left(\left[-h, 0 ; \mathbb{R}^{n}\right]\right.$ denote the family of continuous functions $\phi$ from $[-h, 0]$ to $\mathbb{R}^{n}$ with the norm $\|\phi\|=\sup _{-h \leq \theta \leq 0}|\phi(\theta)|$, where $|\cdot|$ is the Euclidean norm in $\mathbb{R}^{n} ; \mathbb{E}\{\cdot\}$ stands for the mathematical expectation operator. Let $\left(\Omega, \mathcal{F},\left\{\mathcal{F}_{t}\right\}_{t \geq 0}, P\right)$ be a complete probability space with a filtration $\left\{\mathcal{F}_{t}\right\}_{t \geq 0}$ satisfying the usual conditions (i.e., the filtration contains all $P$-null sets and is right continuous). Denote 
by $L_{\mathcal{F}_{0}}^{p}\left([-h, 0] ; \mathbb{R}^{n}\right)$ the family of all $\mathcal{F}_{0}$-measurable $C\left([-h, 0] ; \mathbb{R}^{n}\right)$-valued random variables $\xi=\{\xi(\theta):-h \leq \theta \leq 0\}$ such that $\sup _{-h \leq \theta \leq 0} \mathbb{E}|\xi(\theta)|^{p}<\infty$.

\section{Model Description}

In this paper, we will consider the following genetic regulatory networks [25]:

$$
\begin{gathered}
\dot{m}(t)=-A m(t)+B f(p(t-\sigma(t)))+L, \\
\dot{p}(t)=-C p(t)+D m(t-\tau(t)),
\end{gathered}
$$

where $m(t)=\left[m_{1}(t), m_{2}(t), \ldots, m_{n}(t)\right]^{T}, p(t)=\left[p_{1}(t), p_{2}(t), \ldots, p_{n}(t)\right]^{T}$, and $m_{i}(t)$ and $p_{i}(t)$ are the concentrations of mRNA and protein of the $i$ th node at time $t$, respectively; $A=$ $\operatorname{diag}\left(a_{1}, a_{2}, \ldots, a_{n}\right)$ and $C=\operatorname{diag}\left(c_{1}, c_{2}, \ldots, c_{n}\right)$ denote the degradation or dilution rates of mRNAs and proteins, $D=\operatorname{diag}\left(d_{1}, d_{2}, \ldots, d_{n}\right)$ represents the translation rate, and $B=\left(b_{i j}\right) \in$ $\mathbb{R}^{n \times n}$ is defined as follows:

$$
b_{i j} \begin{cases}>0 & \text { if transcription } j \text { is an activator of gene } i \\ =0, & \text { if there is no link from node } j \text { to } i \\ <0 & \text { if transcription } j \text { is an repressor of gene } i\end{cases}
$$

$f(\cdot)$ denotes the feedback regulation of the protein on the transcription, which is the monotonic function in Hill form, $f_{i}(x)=x^{h_{i}} /\left(1+x^{h_{i}}\right)$, and $h_{i}$ is the Hill coefficient; $\tau(t)$ and $\sigma(t)$ are the time delays; $L=\left[l_{1}, l_{2}, \ldots, l_{n}\right]^{T}, l_{i}$ is the base transcriptional rate of the repressor of gene $i$. Assume $m^{\star}$ and $p^{\star}$ are the equilibrium points of (2.1), defining $x(t)=m(t)-m^{\star}$, $y(t)=p(t)-p^{\star}$, it is easy to get

$$
\begin{gathered}
\dot{x}(t)=-A x(t)+B g(y(t-\sigma(t))), \\
\dot{y}(t)=-C y(t)+D x(t-\tau(t)),
\end{gathered}
$$

where $g(y(t))=f\left(y(t)+P^{\star}\right)-f\left(p^{\star}\right)$, from the definition of $g$, it is easy to get

$$
g(x)(g(x)-K x) \leq 0 .
$$

Taking the Markovian jumping parameters and stochastic delays into account, a Markovian jumping genetic regulatory networks model with mode-dependent delays is considered as

$$
\begin{gathered}
\dot{x}(t)=-A(r(t)) x(t)+B(r(t)) g\left(y\left(t-\sigma_{r(t)}(t)\right)\right), \\
\dot{y}(t)=-C(r(t)) y(t)+D(r(t)) x\left(t-\tau_{r(t)}(t)\right),
\end{gathered}
$$


where $r(t)$ is a continuous-time Markovian process with right continuous trajectories and taking values in a finite set $S=\{1,2, \ldots, N\}$ with the following transition probabilities:

$$
P\{r(t+\Delta t)=j: r(t)=i\}= \begin{cases}\gamma_{i j} \Delta t+O(\Delta t) & \text { if } j \neq i, \\ 1+\gamma_{i j} \Delta t+O(\Delta t) & \text { if } j=i,\end{cases}
$$

where $\Delta t>0$ and $\lim _{\Delta t \rightarrow 0} O(\Delta t) / \Delta t=0$. Here, $\gamma_{i j} \geq 0$ is the transition rate from $i$ to $j$ if $i \neq j$, while $\gamma_{i i}=-\sum_{j=1, j \neq i}^{N} \gamma_{i j}$.

$\tau_{r(t)}(t)$ and $\sigma_{r(t)}(t)$ are the time-varying delays when the mode is in $r(t)$ and we assume that they satisfy the following conditions

$$
0 \leq d_{1 i} \leq \tau_{i}(t) \leq d_{2 i}, \quad \dot{\tau}_{i}(t) \leq h_{i}, \quad 0 \leq e_{1 i} \leq \sigma_{i}(t) \leq e_{2 i}, \quad \dot{\sigma}_{i}(t) \leq \mu_{i},
$$

where $d_{1 i}, d_{2 i}, e_{1 i}, e_{2 i}, h_{i}$, and $\mu_{i}$ are known real constants, for any $i \in S$, denote

$$
\begin{array}{ll}
d_{1}=\min \left\{d_{1 i}, i \in S\right\}, \quad & d_{2}=\max \left\{d_{2 i}, i \in S\right\}, \quad e_{1}=\min \left\{e_{1 i}, i \in S\right\}, \\
& e_{2}=\max \left\{e_{2 i}, i \in S\right\} .
\end{array}
$$

Remark 2.1. In [25], $h_{i}$ and $\mu_{i}$ are assumed to be less than 1. But in practice, they are not always less than 1 . In this paper, we develop the criteria without this restrict. In the following we will give some lemmas, which will play an indispensable role in deriving our criteria.

Lemma 2.2 (see [24]). For any vector $x, y \in \mathbb{R}^{n}$ and matrix $Q>0$, one has the following inequality:

$$
2 x^{T} y \leq x^{T} Q x+y^{T} Q^{-1} y .
$$

Lemma 2.3 (see [30]). For any positive definite matrix $M>0$, scalar $\gamma>0$ and vector function $\omega:[0, \gamma] \rightarrow \mathbb{R}^{n}$ such that the integrations concerned are well defined, then the following inequality holds:

$$
\left(\int_{0}^{\gamma} \omega(s) d s\right)^{T} M\left(\int_{0}^{\gamma} \omega(s) d s\right) \leq r\left(\int_{0}^{\gamma} \omega^{T}(s) M \omega(s) d s\right) .
$$

Lemma 2.4 (see [31]) (Schur complement). Given constant matrices $X, Y$, and $Z$ where $X=X^{T}$ and $0<Y=Y^{T}$. Then $X+Z^{T} Y^{-1} Z<0$ if and only if

$$
\left[\begin{array}{cc}
X & Z^{T} \\
Z & -Y
\end{array}\right]<0, \quad \text { or }\left[\begin{array}{cc}
-Y & Z \\
Z^{T} & X
\end{array}\right]<0
$$

Lemma 2.5 (see [32]). Assume $\Omega, X_{1}$, and $X_{2}$ are constant matrices with appropriate dimensions, $0 \leq \alpha(t) \leq 1$, then

$$
\begin{aligned}
& \Omega-X_{1}<0, \\
& \Omega-X_{2}<0,
\end{aligned}
$$


is equivalent to

$$
\Omega-\alpha(t) X_{1}-(1-\alpha(t)) X_{2}<0
$$

\section{Main Results}

In this section, we first deal with the asymptotical stability problem for the system (2.5). By employing a new Lyapunov-Krasovskii function, some less conservative sufficient criteria for the stability problem of Markovian jumping genetic regulatory networks with modedependent delays are derived in terms of LMIs. Then the results are extended to uncertain case.

Theorem 3.1. The genetic regulatory networks (2.5) is asymptotically stable, if there exist matrix sets $\left\{P_{i}>0, Q_{i}>0, \forall i \in S\right\}$, matrices $N_{j}, M_{j}(j=1,2,3)>0 R_{j}(j=1,2,3,4)>0$, any diagonal positive definite matrix $\Lambda$, and any matrices $U, V$ with appropriate dimensions such that the following LMIs hold:

$$
\begin{aligned}
& \Omega_{1 i}-\epsilon_{1}^{T} R_{1} \epsilon_{1}-\epsilon_{1}^{T} R_{2} \epsilon_{1}<0, \\
& \Omega_{1 i}-\epsilon_{1}^{T} R_{1} \epsilon_{1}-\epsilon_{3}^{T} R_{2} \epsilon_{3}<0, \\
& \Omega_{1 i}-\epsilon_{2}^{T} R_{1} \epsilon_{2}-\epsilon_{1}^{T} R_{2} \epsilon_{1}<0, \\
& \Omega_{1 i}-\epsilon_{2}^{T} R_{1} \epsilon_{2}-\epsilon_{3}^{T} R_{2} \epsilon_{3}<0, \\
& \Omega_{2 i}-\epsilon_{4}^{T} R_{3} \epsilon_{4}-\epsilon_{4}^{T} R_{4} \epsilon_{4}<0, \\
& \Omega_{2 i}-\epsilon_{4}^{T} R_{3} \epsilon_{4}-\epsilon_{6}^{T} R_{4} \epsilon_{6}<0, \\
& \Omega_{2 i}-\epsilon_{5}^{T} R_{3} \epsilon_{5}-\epsilon_{4}^{T} R_{4} \epsilon_{4}<0, \\
& \Omega_{2 i}-\epsilon_{5}^{T} R_{3} \epsilon_{5}-\epsilon_{6}^{T} R_{4} \epsilon_{6}<0,
\end{aligned}
$$

where

$$
\begin{aligned}
& \epsilon_{1}=\left[\begin{array}{lllll}
0 & I & 0 & -I & 0
\end{array}\right], \quad \epsilon_{2}=\left[\begin{array}{lllll}
I & -I & 0 & 0 & 0
\end{array}\right], \quad \epsilon_{3}=\left[\begin{array}{lllll}
0 & -I & I & 0 & 0
\end{array}\right], \\
& \epsilon_{4}=\left[\begin{array}{llllll}
0 & I & 0 & -I & 0 & 0
\end{array}\right], \quad \epsilon_{5}=\left[\begin{array}{llllll}
I & -I & 0 & 0 & 0 & 0
\end{array}\right], \quad \epsilon_{6}=\left[\begin{array}{llllll}
0 & -I & I & 0 & 0 & 0
\end{array}\right], \\
& \Omega_{1 i}=\left[\begin{array}{ccccc}
\Xi_{1} & R_{1} & 0 & 0 & -U A_{i} \\
\star & \Xi_{2} & R_{2} & R_{2}+R_{1}-\frac{d_{1} R_{2}}{d_{2}} & 0 \\
\star & \star & -R_{2}-N_{1} & 0 & 0 \\
\star & \star & \star & -R_{1}-R_{2}-N_{2}+\frac{d_{1} R_{2}}{d_{2}} & 0 \\
\star & \star & \star & \star & \Xi_{3}
\end{array}\right] \text {, }
\end{aligned}
$$




$$
\begin{gathered}
\Omega_{2 i}=\left[\begin{array}{cccccc}
\Pi_{1} & R_{3} & 0 & 0 & -V C_{i} & 0 \\
\star & \Pi_{2} & R_{4} & R_{3}+R_{4}-\frac{e_{1} R_{4}}{e_{2}} & 0 & \Lambda \\
\star & \star & -R_{4}-M_{1} & 0 & 0 & 0 \\
\star & \star & \star & -R_{3}-R_{4}-M_{2}+\frac{e_{1} R_{4}}{e_{2}} & 0 & 0 \\
\star & \star & \star & \star & \Pi_{3} & 0 \\
\star & \star & \star & \star & \Pi_{4}
\end{array}\right], \\
\Xi_{1}=-P_{i} A_{i}-A_{i}^{T} P_{i}+\sum_{j=1}^{N} \gamma_{i j} P_{j}+\sum_{j=1}^{3} N_{j}-\gamma_{i i}\left(d_{2}-d_{1}\right) N_{3}-R_{1}+P_{i}, \\
\Xi_{2}=-\left(1-h_{i}\right) N_{3}-2 R_{1}-2 R_{2}+D_{i}^{T} V D_{i}+D_{i}^{T} Q_{i} D_{i}+\frac{d_{1} R_{2}}{d_{2}}, \\
\Xi_{3}=-U+d_{2}^{2} R_{1}+\left(d_{2}-d_{1}\right)^{2} R_{2}, \\
\Pi_{1}=-Q_{i} C_{i}-C_{i}^{T} Q_{i}+\sum_{j=1}^{N} \gamma_{i j} Q_{j}+\sum_{j=1}^{3} M_{j}-\gamma_{i i}\left(e_{2}-e_{1}\right) M_{3}-R_{3}+Q_{i}, \\
\Pi_{2}=-\left(1-\mu_{i}\right) M_{3}-2 R_{3}-2 R_{4}+\frac{e_{1} R_{4}}{e_{2}} \\
\Pi_{3}=-V+e_{2}^{2} R_{3}+\left(e_{2}-e_{1}\right)^{2} R_{4},
\end{gathered}
$$

Proof. Choose a Lyapunov-Krasovskii functional candidate:

$$
\begin{aligned}
V(i, t, x(t), y(t))= & V_{1}(i, t, x(t), y(t))+V_{2}(i, t, x(t), y(t)) \\
& +V_{3}(i, t, x(t), y(t))+V_{4}(i, t, x(t), y(t)),
\end{aligned}
$$

where

$$
\begin{gathered}
V_{1}(i, t, x(t), y(t))=x^{T}(t) P_{i} x(t)+y^{T}(t) Q_{i} y(t), \\
V_{2}(i, t, x(t), y(t))=\sum_{j=1}^{2} \int_{t-d_{j}}^{t} x^{T}(s) N_{j} x(s) d s+\sum_{j=1}^{2} \int_{t-e_{j}}^{t} y^{T}(s) M_{j} y(s) d s \\
+\int_{t-\tau_{i}(t)}^{t} x^{T}(s) N_{3} x(s) d s+\int_{t-\sigma_{i}(t)}^{t} y^{T}(s) M_{3} y(s) d s,
\end{gathered}
$$




$$
\begin{aligned}
V_{3}(i, t, x(t), y(t)) & =-\gamma_{i i} \int_{-d_{2}}^{-d_{1}} \int_{t+\theta}^{t} x^{T}(s) N_{3} x(s) d s d \theta-\gamma_{i i} \int_{-e_{2}}^{-e_{1}} \int_{t+\theta}^{t} y^{T}(s) M_{3} y(s) d s d \theta, \\
V_{4}(i, t, x(t), y(t))= & d_{2} \int_{-d_{2}}^{0} \int_{t+\theta}^{t} \dot{x}^{T}(s) R_{1} \dot{x}(s) d s d \theta+\left(d_{2}-d_{1}\right) \int_{-d_{2}}^{-d_{1}} \int_{t+\theta}^{t} \dot{x}^{T}(s) R_{2} \dot{x}(s) d s d \theta \\
& +e_{2} \int_{-e_{2}}^{0} \int_{t+\theta}^{t} \dot{y}^{T}(s) R_{3} \dot{y}(s) d s d \theta+\left(e_{2}-e_{1}\right) \int_{-e_{2}}^{-e_{1}} \int_{t+\theta}^{t} \dot{y}^{T}(s) R_{4} \dot{y}(s) d s d \theta .
\end{aligned}
$$

Let $\mathcal{L}$ be the weak infinite generator. Then for each $r(t)=i, i \in S$ along the trajectory of (2.5) one has

$$
\begin{aligned}
\mathcal{L} V_{1}(i, t, x(t), y(t))= & 2 x^{T}(t) P_{i}\left(-A_{i} x(t)+B_{i} g\left(y\left(t-\sigma_{i}(t)\right)\right)+\sum_{j=1}^{N} \gamma_{i j} x^{T}(t) P_{j} x(t)\right. \\
& +2 y^{T}(t) Q_{i}\left(-C_{i} y(t)+D_{i} x\left(t-\tau_{i}(t)\right)\right)+\sum_{j=1}^{N} \gamma_{i j} y^{T}(t) Q_{j} y(t), \\
\mathcal{L} V_{2}(i, t, x(t), y(t)) \leq & \sum_{j=1}^{3} x^{T}(t) N_{j} x(t)-\left(1-h_{i}\right) x^{T}\left(t-\tau_{i}(t)\right) N_{3} x\left(t-\tau_{i}(t)\right) \\
& +\sum_{j=1}^{3} y^{T}(t) M_{j} y(t)-\left(1-\mu_{i}\right) y^{T}\left(t-\sigma_{i}(t)\right) M_{3} y\left(t-\sigma_{i}(t)\right) \\
& -\sum_{j=1}^{2} x^{T}\left(t-d_{j}\right) N_{j} x\left(t-d_{j}\right)-\sum_{j=1}^{2} y^{T}\left(t-e_{j}\right) M_{j} y\left(t-e_{j}\right) \\
& +\sum_{j=1}^{N} \gamma_{i j} \int_{t-\tau_{j}(t)}^{t} x^{T}(s) N_{3} x(s) d s+\sum_{j=1}^{N} \gamma_{i j} \int_{t-\sigma_{j}(t)}^{t} y^{T}(s) M_{3} y(s) d s, \\
\sum_{j=1}^{N} \gamma_{i j} \int_{t-\tau_{j}(t)}^{t} x^{T}(s) N_{3} x(s) d s= & \gamma_{i i} \int_{t-\tau_{i}(t)}^{t} x^{T}(s) N_{3} x(s) d s+\sum_{j \neq i} \gamma_{i j} \int_{t-\tau_{j}(t)}^{t} x^{T}(s) N_{3} x(s) d s \\
\leq & \gamma_{i i} \int_{t-d_{1}}^{t} x^{T}(s) N_{3} x(s) d s+\sum_{j \neq i} \gamma_{i j} \int_{t-d_{2}}^{t} x^{T}(s) N_{3} x(s) d s \\
= & \gamma_{i i} \int_{t-d_{1}}^{t} x^{T}(s) N_{3} x(s) d s-\gamma_{i i} \int_{t-d_{2}}^{t} x^{T}(s) N_{3} x(s) d s \\
= & -\gamma_{i i} \int_{t-d_{2}}^{t-d_{1}} x^{T}(s) N_{3} x(s) d s .
\end{aligned}
$$


Similarly

$$
\begin{aligned}
& \sum_{j=1}^{N} \gamma_{i j} \int_{t-\sigma_{j}(t)}^{t} y^{T}(s) M_{3} y(s) d s \leq-\gamma_{i i} \int_{t-e_{2}}^{t-e_{1}} y^{T}(s) M_{3} y(s) d s \\
& \mathcal{L} V_{3}(i, t, x(t), y(t))=-\gamma_{i i}\left(d_{2}-d_{1}\right) x^{T}(t) N_{3} x(t)+\gamma_{i i} \int_{t-d_{2}}^{t-d_{1}} x^{T}(s) N_{3} x(s) d s \\
& -\gamma_{i i}\left(e_{2}-e_{1}\right) y^{T}(t) M_{3} y(t)+\gamma_{i i} \int_{t-e_{2}}^{t-e_{1}} y^{T}(s) M_{3} y(s) d s, \\
& \mathcal{L} V_{4}(i, t, x(t), y(t))=d_{2}^{2} \dot{x}^{T}(t) R_{1} \dot{x}(t)-d_{2} \int_{t-d_{2}}^{t} \dot{x}^{T}(s) R_{1} \dot{x}(s) d s+\left(d_{2}-d_{1}\right)^{2} \dot{x}^{T}(t) R_{2} \dot{x}(t) \\
& +\left(e_{2}-e_{1}\right)^{2} \dot{y}^{T}(t) R_{4} \dot{y}(t) d s-e_{2} \int_{t-e_{2}}^{t} \dot{y}^{T}(s) R_{3} \dot{y}(s) d s+e_{2}^{2} \dot{y}^{T}(t) R_{3} \dot{y}(t) \\
& -\left(d_{2}-d_{1}\right) \int_{t-d_{2}}^{t-d_{1}} \dot{x}^{T}(s) R_{2} \dot{x}(s) d s-\left(e_{2}-e_{1}\right) \int_{t-e_{2}}^{t-e_{1}} \dot{y}^{T}(s) R_{4} \dot{y}(s) d s .
\end{aligned}
$$

Note that

$$
\begin{aligned}
-d_{2} \int_{t-d_{2}}^{t} \dot{x}^{T}(s) R_{1} \dot{x}(s) d s \\
=-d_{2} \int_{t-d_{2}}^{t-\tau_{i}(t)} \dot{x}^{T}(s) R_{1} \dot{x}(s) d s-d_{2} \int_{t-\tau_{i}(t)}^{t} \dot{x}^{T}(s) R_{1} \dot{x}(s) d s \\
=-\left(d_{2}-\tau_{i}(t)\right) \int_{t-d_{2}}^{t-\tau_{i}(t)} \dot{x}^{T}(s) R_{1} \dot{x}(s) d s-\tau_{i}(t) \int_{t-d_{2}}^{t-\tau_{i}(t)} \dot{x}^{T}(s) R_{1} \dot{x}(s) d s \\
\quad-\tau_{i}(t) \int_{t-\tau_{i}(t)}^{t} \dot{x}^{T}(s) R_{1} \dot{x}(s) d s-\left(d_{2}-\tau_{i}(t)\right) \int_{t-\tau_{i}(t)}^{t} \dot{x}^{T}(s) R_{1} \dot{x}(s) d s \\
\leq-x^{T}\left(t-\tau_{i}(t)\right) R_{1} x\left(t-\tau_{i}(t)\right)+2 x^{T}\left(t-\tau_{i}(t)\right) R_{1} x\left(t-d_{2}\right)-x^{T}\left(t-d_{2}\right) R_{1} x\left(t-d_{2}\right) \\
\quad-x^{T}(t) R_{1} x(t)+2 x^{T}(t) R_{1} x\left(t-\tau_{i}(t)\right)-x^{T}\left(t-\tau_{i}(t)\right) R_{1} x\left(t-\tau_{i}(t)\right) \\
\quad-\left[\frac{\tau_{i}(t)}{d_{2}-\tau_{i}(t)}\right]\left[x^{T}\left(t-\tau_{i}(t)\right)-x^{T}\left(t-d_{2}\right)\right] R_{1}\left[x\left(t-\tau_{i}(t)\right)-x\left(t-d_{2}\right)\right] \\
\quad-\left[\frac{d_{2}-\tau_{i}(t)}{\tau_{i}(t)}\right]\left[x^{T}(t)-x^{T}\left(t-\tau_{i}(t)\right)\right] R_{1}\left[x(t)-x\left(t-\tau_{i}(t)\right)\right] \\
\leq-x^{T}\left(t-\tau_{i}(t)\right) R_{1} x\left(t-\tau_{i}(t)\right)+2 x^{T}\left(t-\tau_{i}(t)\right) R_{1} x\left(t-d_{2}\right)-x^{T}\left(t-d_{2}\right) R_{1} x\left(t-d_{2}\right) \\
\quad-x^{T}(t) R_{1} x(t)+2 x^{T}(t) R_{1} x\left(t-\tau_{i}(t)\right)-x^{T}\left(t-\tau_{i}(t)\right) R_{1} x\left(t-\tau_{i}(t)\right)
\end{aligned}
$$


Mathematical Problems in Engineering

$$
\begin{aligned}
& -\left(\frac{\tau_{i}(t)}{d_{2}}\right)\left[x^{T}\left(t-\tau_{i}(t)\right)-x^{T}\left(t-d_{2}\right)\right] R_{1}\left[x\left(t-\tau_{i}(t)\right)-x\left(t-d_{2}\right)\right] \\
& -\left[1-\left(\frac{\tau_{i}(t)}{d_{2}}\right)\right]\left[x^{T}(t)-x^{T}\left(t-\tau_{i}(t)\right)\right] R_{1}\left[x(t)-x\left(t-\tau_{i}(t)\right)\right] .
\end{aligned}
$$

Similarly,

$$
\begin{aligned}
-\left(d_{2}-\right. & \left.d_{1}\right) \int_{t-d_{2}}^{t-d_{1}} \dot{x}^{T}(s) R_{2} \dot{x}(s) d s \\
\leq & -x^{T}\left(t-\tau_{i}(t)\right) R_{2} x\left(t-\tau_{i}(t)\right)+2 x^{T}\left(t-\tau_{i}(t)\right) R_{2} x\left(t-d_{2}\right)-x^{T}\left(t-d_{2}\right) R_{2} x\left(t-d_{2}\right) \\
& -x^{T}\left(t-d_{1}\right) R_{2} x\left(t-d_{1}\right)+2 x^{T}\left(t-d_{1}\right) R_{2} x\left(t-\tau_{i}(t)\right)-x^{T}\left(t-\tau_{i}(t)\right) R_{2} x\left(t-\tau_{i}(t)\right) \\
& +\left(\frac{d_{1}}{d_{2}}\right)\left[x^{T}\left(t-\tau_{i}(t)\right)-x^{T}\left(t-d_{2}\right)\right] R_{2}\left[x\left(t-\tau_{i}(t)\right)-x\left(t-d_{2}\right)\right] \\
& -\left(\frac{\tau_{i}(t)}{d_{2}}\right)\left[x^{T}\left(t-\tau_{i}(t)\right)-x^{T}\left(t-d_{2}\right)\right] R_{2}\left[x\left(t-\tau_{i}(t)\right)-x\left(t-d_{2}\right)\right] \\
& -\left[1-\left(\frac{\tau_{i}(t)}{d_{2}}\right)\right]\left[x^{T}\left(t-d_{1}\right)-x^{T}\left(t-\tau_{i}(t)\right)\right] R_{2}\left[x\left(t-d_{1}\right)-x\left(t-\tau_{i}(t)\right)\right], \\
\left.-e_{2}\right]_{t-e_{2}}^{t} & \dot{y}^{T}(s) R_{3} \dot{y}(s) d s \\
\leq & -y^{T}\left(t-\sigma_{i}(t)\right) R_{3} y\left(t-\sigma_{i}(t)\right)+2 y^{T}\left(t-\sigma_{i}(t)\right) R_{3} y\left(t-e_{2}\right)-y^{T}\left(t-e_{2}\right) R_{3} y\left(t-e_{2}\right) \\
& -y^{T}(t) R_{3} y(t)+2 y^{T}(t) R_{3} y\left(t-\sigma_{i}(t)\right)-y^{T}\left(t-\sigma_{i}(t)\right) R_{3} y\left(t-\sigma_{i}(t)\right) \\
& -\left[\frac{\sigma_{i}(t)}{e_{2}}\right]\left[y^{T}\left(t-\sigma_{i}(t)\right)-y^{T}\left(t-e_{2}\right)\right] R_{3}\left[y\left(t-\sigma_{i}(t)\right)-y\left(t-e_{2}\right)\right] \\
& -\left[1-\left(\frac{\sigma_{i}(t)}{e_{2}}\right)\right]\left[y^{T}(t)-y^{T}\left(t-\sigma_{i}(t)\right)\right] R_{3}\left[y(t)-y\left(t-\sigma_{i}(t)\right)\right] \\
& -\left(\frac{\sigma_{i}(t)}{e_{2}}\right)\left[y^{T}\left(t-\sigma_{i}(t)\right)-y^{T}\left(t-e_{2}\right)\right] R_{4}\left[y\left(t-\sigma_{i}(t)\right)-y\left(t-e_{2}\right)\right] \\
- & -y^{T}\left(t-\sigma_{i}(t)\right) R_{4} y\left(t-\sigma_{i}(t)\right)+2 y^{T}\left(t-\sigma_{i}(t)\right) R_{4} y\left(t-e_{2}\right)-y^{T}\left(t-e_{2}\right) R_{4} y\left(t-e_{2}\right) \\
& -y^{T}\left(t-e_{1}\right) R_{4} y\left(t-e_{1}\right)+2 y^{T}\left(t-e_{1}\right) R_{4} y\left(t-\sigma_{i}(t)\right)-y^{T}\left(t-\sigma_{i}(t)\right) R_{4} y\left(t-\sigma_{i}(t)\right) \\
-\left(e_{2}-e_{1}\right) \int_{t-e_{2}}^{t-e_{1}} \dot{y}^{T}(s) R_{4} \dot{y}(s) d s & \left(y^{T}\left(t-e_{1}\right)-y^{T}\left(t-\sigma_{i}(t)\right)\right] R_{4}\left[y\left(t-e_{1}\right)-y\left(t-\sigma_{i}(t)\right)\right] . \\
& {\left.\left[e_{i}(t)\right)-y^{T}\left(t-e_{2}\right)\right] R_{4}\left[y\left(t-\sigma_{i}(t)\right)-y\left(t-e_{2}\right)\right] } \\
& \\
&
\end{aligned}
$$


Noting the sector condition (2.4), for any positive matrix $\Lambda$ we have

$$
2 y^{T}\left(t-\sigma_{i}(t)\right) \Lambda g\left(y\left(t-\sigma_{i}(t)\right)\right)-2 g^{T}\left(y\left(t-\sigma_{i}(t)\right)\right) \Lambda K^{-1} g\left(y\left(t-\sigma_{i}(t)\right)\right) \geq 0
$$

For any matrices $U$ and $V$ with appropriate dimensions, we have

$$
\begin{gathered}
-2 \dot{x}^{T}(t) U\left[\dot{x}(t)+A_{i} x(t)-B_{i} g\left(y\left(t-\sigma_{i}(t)\right)\right)\right]=0, \\
-2 \dot{y}^{T}(t) V\left[\dot{y}(t)+C_{i} y(t)-D_{i} x\left(t-\tau_{i}(t)\right)\right]=0 .
\end{gathered}
$$

By Lemma 2.3 we can get the following inequalities:

$$
\begin{gathered}
2 x^{T}(t) P_{i} B_{i} g\left(y\left(t-\sigma_{i}(t)\right)\right) \leq x^{T}(t) P_{i} x(t)+g^{T}\left(y\left(t-\sigma_{i}(t)\right)\right) B_{i}^{T} P_{i} B_{i} g\left(y\left(t-\sigma_{i}(t)\right)\right), \\
2 y^{T}(t) Q_{i} D_{i} x\left(t-\tau_{i}(t)\right) \leq y^{T}(t) Q_{i} y(t)+x^{T}\left(t-\tau_{i}(t)\right) D_{i}^{T} Q_{i} D_{i} x\left(t-\tau_{i}(t)\right), \\
2 \dot{x}^{T}(t) U B_{i} g\left(y\left(t-\sigma_{i}(t)\right)\right) \leq \dot{x}^{T}(t) U \dot{x}(t)+g^{T}\left(y\left(t-\sigma_{i}(t)\right)\right) B_{i}^{T} U B_{i} g\left(y\left(t-\sigma_{i}(t)\right)\right), \\
2 \dot{y}^{T}(t) V D_{i} x\left(t-\tau_{i}(t)\right) \leq \dot{y}^{T}(t) V \dot{y}(t)+x^{T}\left(t-\tau_{i}(t)\right) D_{i}^{T} V D_{i} x\left(t-\tau_{i}(t)\right) .
\end{gathered}
$$

From (3.3) to (3.11) we can get

$$
\begin{aligned}
\mathcal{L V}(i, t, x(t), y(t)) \leq & \xi^{T} \Omega_{1 i} \xi(t)-\left(\frac{\tau_{i}(t)}{d_{2}}\right) \xi^{T}(t) \epsilon_{1}^{T} R_{1} \epsilon_{1} \xi(t)-\left[1-\left(\frac{\tau_{i}(t)}{d_{2}}\right)\right] \xi^{T}(t) \epsilon_{2}^{T} R_{1} \epsilon_{2} \xi(t) \\
& -\left(\frac{\tau_{i}(t)}{d_{2}}\right) \xi^{T}(t) \epsilon_{1}^{T} R_{2} \epsilon_{1} \xi(t)-\left[1-\left(\frac{\tau_{i}(t)}{d_{2}}\right)\right] \xi^{T}(t) \epsilon_{3}^{T} R_{2} \epsilon_{3} \xi(t) \\
& +\eta^{T} \Omega_{2 i} \eta(t)-\left(\frac{\sigma_{i}(t)}{e_{2}}\right) \eta^{T}(t) \epsilon_{4}^{T} R_{3} \epsilon_{4} \eta(t)-\left[1-\left(\frac{\sigma_{i}(t)}{e_{2}}\right)\right] \eta^{T}(t) \epsilon_{5}^{T} R_{3} \epsilon_{5} \eta(t) \\
& -\left(\frac{\sigma_{i}(t)}{e_{2}}\right) \eta^{T}(t) \epsilon_{4}^{T} R_{4} \epsilon_{4} \eta(t)-\left[1-\left(\frac{\sigma_{i}(t)}{e_{2}}\right)\right] \eta^{T}(t) \epsilon_{6}^{T} R_{4} \epsilon_{6} \eta(t),
\end{aligned}
$$

where

$$
\begin{gathered}
\xi(t)=\left[x^{T}(t), x^{T}\left(t-\tau_{i}(t)\right), x^{T}\left(t-d_{1}\right), x^{T}\left(t-d_{2}\right), \dot{x}^{T}(t)\right]^{T}, \\
\eta(t)=\left[y^{T}(t), y^{T}\left(t-\sigma_{i}(t)\right), y^{T}\left(t-e_{1}\right), y^{T}\left(t-e_{2}\right), \dot{y}^{T}(t), g^{T}\left(y\left(t-\sigma_{i}(t)\right)\right)\right]^{T} .
\end{gathered}
$$

By Lemma 2.5, (3.12) < 0 is equivalent to (3.1). Then by the Lyapunov-Krasovskii stability theorem that the genetic regulatory networks (2.5) is asymptotically stable in the mean square. Hence, this completes the proof. 
In the proof of Theorem 3.1, if we deal with the terms $-d_{2} \int_{t-d_{2}}^{t} \dot{x}^{T}(s) R_{1} \dot{x}(s) d s,-\left(d_{2}-\right.$ $\left.d_{1}\right) \int_{t-d_{2}}^{t-d_{1}} \dot{x}^{T}(s) R_{2} \dot{x}(s) d s,-e_{2} \int_{t-e_{2}}^{t} \dot{y}^{T}(s) R_{3} \dot{y}(s) d s,-\left(e_{2}-e_{1}\right) \int_{t-e_{2}}^{t-e_{1}} \dot{y}^{T}(s) R_{4} \dot{y}(s) d s$ as follows:

$$
\begin{aligned}
& -d_{2} \int_{t-d_{2}}^{t} \dot{x}^{T}(s) R_{1} \dot{x}(s) d s \\
& \quad \leq-x^{T}\left(t-\tau_{i}(t)\right) R_{1} x\left(t-\tau_{i}(t)\right)+2 x^{T}\left(t-\tau_{i}(t)\right) R_{1} x\left(t-d_{2}\right)-x^{T}\left(t-d_{2}\right) R_{1} x\left(t-d_{2}\right) \\
& \quad-x^{T}(t) R_{1} x(t)+2 x^{T}(t) R_{1} x\left(t-\tau_{i}(t)\right)-x^{T}\left(t-\tau_{i}(t)\right) R_{1} x\left(t-\tau_{i}(t)\right), \\
& -\left(d_{2}-d_{1}\right) \int_{t-d_{2}}^{t-d_{1}} \dot{x}^{T}(s) R_{2} \dot{x}(s) d s \\
& \quad \leq-x^{T}\left(t-\tau_{i}(t)\right) R_{2} x\left(t-\tau_{i}(t)\right)+2 x^{T}\left(t-\tau_{i}(t)\right) R_{2} x\left(t-d_{2}\right)-x^{T}\left(t-d_{2}\right) R_{2} x\left(t-d_{2}\right) \\
& \quad-x^{T}\left(t-d_{1}\right) R_{2} x\left(t-d_{1}\right)+2 x^{T}\left(t-d_{1}\right) R_{2} x\left(t-\tau_{i}(t)\right)-x^{T}\left(t-\tau_{i}(t)\right) R_{2} x\left(t-\tau_{i}(t)\right), \\
& -e_{2} \int_{t-e_{2}}^{t} \dot{y}^{T}(s) R_{3} \dot{y}(s) d s \\
& \quad \leq-y^{T}\left(t-\sigma_{i}(t)\right) R_{3} y\left(t-\sigma_{i}(t)\right)+2 y^{T}\left(t-\sigma_{i}(t)\right) R_{3} y\left(t-e_{2}\right)-y^{T}\left(t-e_{2}\right) R_{3} y\left(t-e_{2}\right) \\
& \quad-y^{T}(t) R_{3} y(t)+2 y^{T}(t) R_{3} y\left(t-\sigma_{i}(t)\right)-y^{T}\left(t-\sigma_{i}(t)\right) R_{3} y\left(t-\sigma_{i}(t)\right), \\
& \quad-\left(e_{2}-e_{1}\right) \int_{t-e_{2}}^{t-e_{1}} \dot{y}^{T}(s) R_{4} \dot{y}(s) d s \\
& \quad \leq-y^{T}\left(t-\sigma_{i}(t)\right) R_{4} y\left(t-\sigma_{i}(t)\right)+2 y^{T}\left(t-\sigma_{i}(t)\right) R_{4} y\left(t-e_{2}\right)-y^{T}\left(t-e_{2}\right) R_{4} y\left(t-e_{2}\right) \\
& \quad-y^{T}\left(t-e_{1}\right) R_{4} y\left(t-e_{1}\right)+2 y^{T}\left(t-e_{1}\right) R_{4} y\left(t-\sigma_{i}(t)\right)-y^{T}\left(t-\sigma_{i}(t)\right) R_{4} y\left(t-\sigma_{i}(t)\right)
\end{aligned}
$$

that is, we do not use Lemma 2.5 , then we will have the following corollary.

Corollary 3.2. The genetic regulatory networks (2.5) is asymptotically stable, if there exist matrix sets $\left\{P_{i}>0, Q_{i}>0, \forall i \in S\right\}$, matrices $N_{j}, M_{j}(j=1,2,3)>0 R_{j}(j=1,2,3,4)>0$, any diagonal positive definite matrix $\Lambda$, and any matrices $U$ and $V$ with appropriate dimensions such that the following LMIs hold:

$$
\Omega_{1 i}<0, \quad \Omega_{2 i}<0,
$$

where, $\Omega_{1 i}$ and $\Omega_{2 i}$ are defined in Theorem 3.1.

Remark 3.3. In the proof of Theorem 3.1, if we ignore the terms $-\left(\tau_{i}(t) / d_{2}\right) \xi^{T}(t) \epsilon_{1}^{T} R_{1} \epsilon_{1} \xi(t)$, $-\left[1-\left(\tau_{i}(t) / d_{2}\right)\right] \xi^{T}(t) \epsilon_{2}^{T} R_{1} \epsilon_{2} \xi(t)$, and $-\left(\sigma_{i}(t) / e_{2}\right) \eta^{T}(t) \epsilon_{4}^{T} R_{3} \epsilon_{4} \eta(t),-\left[1-\left(\sigma_{i}(t) / e_{2}\right)\right] \eta^{T}(t) \epsilon_{5}^{T}$ $R_{3} \epsilon_{5} \eta(t)$, we can also get sufficient conditions ensuring the robust stability of the genetic regulatory networks. But the conditions are conservative to some extent. By considering the terms $-\left(\tau_{i}(t) / d_{2}\right) \xi^{T}(t) \epsilon_{1}^{T} R_{1} \epsilon_{1} \xi(t)$, and $-\left[1-\left(\tau_{i}(t) / d_{2}\right)\right] \xi^{T}(t) \epsilon_{2}^{T} R_{1} \epsilon_{2} \xi(t),-\left(\sigma_{i}(t) / e_{2}\right) \eta^{T}(t) \epsilon_{4}^{T} R_{3} \epsilon_{4} \eta(t)$, $-\left[1-\left(\sigma_{i}(t) / e_{2}\right)\right] \eta^{T}(t) \epsilon_{5}^{T} R_{3} \epsilon_{5} \eta(t)$, we can get a less conservative criterion. The illustrate examples will show this in Section 4. 
In the following, we will extend our results to uncertain case. We consider the following Markovian jumping genetic regulatory networks with mode-dependent delays and parameter uncertainties:

$$
\begin{gathered}
\dot{x}(t)=-\left(A_{i}+\Delta A_{i}\right) x(t)+\left(B_{i}+\Delta B_{i}\right) g\left(y\left(t-\sigma_{i}(t)\right)\right), \\
\dot{y}(t)=-\left(C_{i}+\Delta C_{i}\right) y(t)+\left(D_{i}+\Delta D_{i}\right) x\left(t-\tau_{i}(t)\right),
\end{gathered}
$$

where $\Delta A_{i}, \Delta B_{i}, \Delta C_{i}$, and $\Delta D_{i}$ are the parametric uncertainties satisfying:

$$
\left[\Delta A_{i}, \Delta B_{i}, \Delta C_{i}, \Delta D_{i}\right]=E_{i} F_{i}\left[H_{a i}, H_{b i}, H_{c i}, H_{d i}\right] .
$$

$E_{i}, H_{a i}, H_{b i}, H_{c i}$ and $H_{d i}$ are the known real constant matrices with appropriate dimensions, $F_{i}$ satisfies

$$
F_{i}^{T} F_{i} \leq I, \quad i \in S
$$

Theorem 3.4. The genetic regulatory networks (3.16) is robust asymptotically stable, if there exist $P_{1}>0, P_{2}>0, \ldots, P_{n}>0, Q_{1}>0, Q_{2}>0, \ldots, Q_{n}>0, N_{j}, M_{j}(j=1,2,3)>0, R_{j}(j=1,2,3,4)>$ 0 , real number $\left\{\varepsilon_{i}, i \in S\right\}$, any diagonal positive definite matrix $\Lambda$, and any matrices $U$ and $V$ with appropriate dimensions such that the following LMIs hold:

$$
\begin{aligned}
& \bar{\Omega}_{1 i}-\epsilon_{1}^{T} R_{1} \epsilon_{1}-\epsilon_{1}^{T} R_{2} \epsilon_{1}<0, \\
& \bar{\Omega}_{1 i}-\epsilon_{1}^{T} R_{1} \epsilon_{1}-\epsilon_{3}^{T} R_{2} \epsilon_{3}<0, \\
& \bar{\Omega}_{1 i}-\epsilon_{2}^{T} R_{1} \epsilon_{2}-\epsilon_{1}^{T} R_{2} \epsilon_{1}<0, \\
& \bar{\Omega}_{1 i}-\epsilon_{2}^{T} R_{1} \epsilon_{2}-\epsilon_{3}^{T} R_{2} \epsilon_{3}<0, \\
& \bar{\Omega}_{2 i}-\epsilon_{4}^{T} R_{3} \epsilon_{4}-\epsilon_{4}^{T} R_{4} \epsilon_{4}<0, \\
& \bar{\Omega}_{2 i}-\epsilon_{4}^{T} R_{3} \epsilon_{4}-\epsilon_{6}^{T} R_{4} \epsilon_{6}<0, \\
& \bar{\Omega}_{2 i}-\epsilon_{5}^{T} R_{3} \epsilon_{5}-\epsilon_{4}^{T} R_{4} \epsilon_{4}<0, \\
& \bar{\Omega}_{2 i}-\epsilon_{5}^{T} R_{3} \epsilon_{5}-\epsilon_{6}^{T} R_{4} \epsilon_{6}<0,
\end{aligned}
$$

where

$$
\begin{aligned}
& \overline{\Omega_{1 i}}=\left[\begin{array}{cc}
\bar{\Gamma}_{1 i} & \phi_{1 i} \\
\star & \psi_{1 i}
\end{array}\right], \quad \bar{\Omega}_{2 i}=\left[\begin{array}{cc}
\bar{\Gamma}_{2 i} & \phi_{2 i} \\
\star & \varphi_{2 i}
\end{array}\right], \\
& \bar{\Gamma}_{1 i}=\left[\begin{array}{ccccc}
\bar{\Xi}_{1} & R_{1} & 0 & 0 & -U A_{i} \\
\star & \bar{\Xi}_{2} & R_{2} & R_{2}+R_{1}-\frac{d_{1} R_{2}}{d_{2}} & 0 \\
\star & \star & -R_{2}-N_{1} & 0 & 0 \\
\star & \star & \star & -R_{1}-R_{2}-N_{2}+\frac{d_{1} R_{2}}{d_{2}} & 0 \\
\star & \star & \star & \star & \bar{\Xi}_{3},
\end{array}\right], \\
& \bar{\Xi}_{1}=-P_{i} A_{i}-A_{i}^{T} P_{i}+\sum_{j=1}^{N} \gamma_{i j} P_{j}+\sum_{j=1}^{3} N_{j}-\gamma_{i i}\left(d_{2}-d_{1}\right) N_{3}-R_{1}+P_{i}+2 \varepsilon_{i} H_{a i}^{T} H_{a i},
\end{aligned}
$$


Mathematical Problems in Engineering

$$
\begin{aligned}
& \bar{\Xi}_{2}=-\left(1-h_{i}\right) N_{3}-2 R_{1}-2 R_{2}+D_{i}^{T} V D_{i}+D_{i}^{T} Q_{i} D_{i}+2 \varepsilon_{i} H_{d i}^{T} H_{d i}+\frac{d_{1} R_{2}}{d_{2}}, \\
& \psi_{1 i}=\operatorname{diag}\left(-\varepsilon_{i} I,-I,-I,-I,-\varepsilon_{i} I\right), \\
& \phi_{1 i}=\left[\begin{array}{ccccc}
\sqrt{2} P_{i} E_{i} & 0 & 0 & 0 & 0 \\
0 & 0 & 0 & 0 & 0 \\
0 & 0 & 0 & 0 & 0 \\
0 & 0 & 0 & 0 & 0 \\
0 & 0 & 0 & 0 & 0 \\
0 & 0 & 0 & 0 & \sqrt{2} U E_{i}
\end{array}\right], \\
& \bar{\Gamma}_{2 i}=\left[\begin{array}{cccccc}
\bar{\Pi}_{1} & R_{3} & 0 & 0 & -V C_{i} & 0 \\
\star & \Pi_{2} & R_{4} & R_{3}+R_{4}-\frac{e_{1} R_{4}}{e_{2}} & 0 & \Lambda \\
\star & \star & -R_{4}-M_{1} & 0 & 0 & 0 \\
\star & \star & \star & -R_{3}-R_{4}-M_{2}+\frac{e_{1} R_{4}}{e_{2}} & 0 & 0 \\
\star & \star & \star & \star & \Pi_{3} & 0 \\
\star & \star & \star & \star & \star & \bar{\Pi}_{4}
\end{array}\right], \\
& \bar{\Pi}_{1}=-Q_{i} C_{i}-C_{i}^{T} Q_{i}+\sum_{j=1}^{N} \gamma_{i j} Q_{j}+\sum_{j=1}^{3} M_{j}-\gamma_{i i}\left(e_{2}-e_{1}\right) M_{3}-R_{3}+Q_{i}+2 \varepsilon_{i} H_{c i}^{T} H_{c i}, \\
& \bar{\Pi}_{4}=-2 \Lambda K^{-1}+B_{i}^{T} U B_{i}+B_{i}^{T} P_{i} B_{i}+2 \varepsilon_{i} H_{b i}^{T} H_{b i} \\
& \phi_{2 i}=\left[\begin{array}{cccccc}
\sqrt{2} Q_{i} E_{i} & 0 & 0 & 0 & 0 & 0 \\
0 & 0 & 0 & 0 & 0 & 0 \\
0 & 0 & 0 & 0 & 0 & 0 \\
0 & 0 & 0 & 0 & 0 & 0 \\
0 & 0 & 0 & 0 & 0 & 0 \\
0 & 0 & 0 & 0 & \sqrt{2} V E_{i} & 0 \\
0 & 0 & 0 & 0 & 0 & 0
\end{array}\right], \\
& \psi_{2 i}=\operatorname{diag}\left(-\varepsilon_{i} I,-I,-I,-I,-I,-\varepsilon_{i} I\right) .
\end{aligned}
$$

Proof. Consider the same Lyapunov-Krasovskii functional (3.3), do the differential along the trajectory (3.16), one can readily get

$$
\begin{aligned}
& \left\llcorner V(i, t, x(t), y(t)) \leq \xi^{T} \overline{\Gamma_{1 i}} \xi(t)-\left(\frac{\tau_{i}(t)}{d_{2}}\right) \xi^{T}(t) \epsilon_{1}^{T} R_{1} \epsilon_{1} \xi(t)-\left[1-\left(\frac{\tau_{i}(t)}{d_{2}}\right)\right] \xi^{T}(t) \epsilon_{2}^{T} R_{1} \epsilon_{2} \xi(t)\right. \\
& -\left(\frac{\tau_{i}(t)}{d_{2}}\right) \xi^{T}(t) \epsilon_{1}^{T} R_{2} \epsilon_{1} \xi(t)-\left[1-\left(\frac{\tau_{i}(t)}{d_{2}}\right)\right] \xi^{T}(t) \epsilon_{3}^{T} R_{2} \epsilon_{3} \xi(t)
\end{aligned}
$$




$$
\begin{aligned}
& +\eta^{T} \overline{\Gamma_{2 i}} \eta(t)-\left(\frac{\sigma_{i}(t)}{e_{2}}\right) \eta^{T}(t) \epsilon_{4}^{T} R_{3} \epsilon_{4} \eta(t)-\left[1-\left(\frac{\sigma_{i}(t)}{e_{2}}\right)\right] \eta^{T}(t) \epsilon_{5}^{T} R_{3} \epsilon_{5} \eta(t) \\
& -\left(\frac{\sigma_{i}(t)}{e_{2}}\right) \eta^{T}(t) \epsilon_{4}^{T} R_{4} \epsilon_{4} \eta(t)-\left[1-\left(\frac{\sigma_{i}(t)}{e_{2}}\right)\right] \eta^{T}(t) \epsilon_{6}^{T} R_{4} \epsilon_{6} \eta(t) \\
& +2 \varepsilon_{i}^{-1} x^{T}(t) P_{i} E_{i} E_{i}^{T} P_{i} x(t)+2 \varepsilon_{i}^{-1} y^{T}(t) Q_{i} E_{i} E_{i}^{T} Q_{i} y(t) \\
& +2 \varepsilon_{i}^{-1} \dot{x}^{T}(t) U E_{i} E_{i}^{T} U x(t)+2 \varepsilon_{i}^{-1} \dot{y}^{T}(t) V E_{i} E_{i}^{T} V \dot{y}(t) .
\end{aligned}
$$

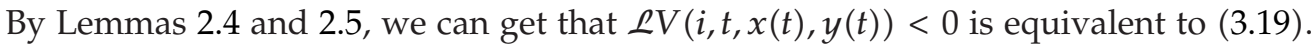
Hence the Markovian jumping genetic regulatory network with mode-dependent delays and parameter uncertainties is robust asymptotically stable. This completes the proof.

As mentioned in Theorem 3.1, if we ignore the terms $-\left(\tau_{i}(t) / d_{2}\right) \xi^{T}(t) \epsilon_{1}^{T} R_{1} \epsilon_{1} \xi(t),-[1-$ $\left.\left(\tau_{i}(t) / d_{2}\right)\right] \xi^{T}(t) \epsilon_{2}^{T} R_{1} \epsilon_{2} \xi(t),-\left(\sigma_{i}(t) / e_{2}\right) \eta^{T}(t) \epsilon_{4}^{T} R_{3} \epsilon_{4} \eta(t)$, and $-\left[1-\left(\sigma_{i}(t) / e_{2}\right)\right] \eta^{T}(t) \epsilon_{5}^{T} R_{3} \epsilon_{5} \eta(t)$, we can get the following corollary.

Corollary 3.5. The genetic regulatory networks (3.16) is robust asymptotically stable, if there exist $P_{1}>0, P_{2}>0, \ldots, P_{n}>0, Q_{1}>0, Q_{2}>0, \ldots, Q_{n}>0, N_{j}, M_{j}(j=1,2,3)>0, R_{j}(j=1,2,3,4)>$ 0 , real number $\left\{\varepsilon_{i}, i \in S\right\}$, any diagonal positive definite matrix $\Lambda$, and any matrices $U$ and $V$ with appropriate dimensions such that the following LMIs hold:

$$
\overline{\Omega_{1 i}}<0, \quad \overline{\Omega_{2 i}}<0,
$$

where $\overline{\Omega_{1 i}}$ and $\overline{\Omega_{2 i}}$ are defined in Theorem 3.4 .

\section{Illustrative Examples}

In this section, two numerical examples are given to illustrate the effectiveness of the derived results.

Example 4.1. Consider (2.5) where

$$
\begin{aligned}
& A_{1}=\left[\begin{array}{ll}
3 & 0 \\
0 & 3
\end{array}\right], \quad B_{1}=\left[\begin{array}{cc}
1 & -2 \\
0.8 & 0
\end{array}\right], \quad C_{1}=\left[\begin{array}{ll}
2 & 0 \\
0 & 2
\end{array}\right], \quad D_{1}=\left[\begin{array}{ll}
1 & 0 \\
0 & 1
\end{array}\right], \\
& A_{2}=\left[\begin{array}{ll}
3 & 0 \\
0 & 3
\end{array}\right], \quad B_{2}=\left[\begin{array}{cc}
-1 & 0 \\
1 & 2
\end{array}\right], \quad C_{2}=\left[\begin{array}{ll}
2 & 0 \\
0 & 2
\end{array}\right], \quad D_{2}=\left[\begin{array}{cc}
-1 & 0 \\
0 & 1
\end{array}\right] \text {. }
\end{aligned}
$$

The nonlinear regulation function is taken as $g(x)=x^{2} /\left(1+x^{2}\right)$, so we can easily get $k_{i}=0.65$, the transmission probability is assumed to be $\gamma=\left[\begin{array}{cc}-0.4 & 0.4 \\ 0.6 & -0.6\end{array}\right]$, and time delays are chosen as

$$
\begin{array}{cl}
\tau_{1}(t)=0.4+0.2 \cos (t), \quad \tau_{2}(t) & =0.4+0.1 \sin (t), \quad \sigma_{1}(t)=0.2+0.1 \sin (t), \\
\sigma_{2}(t) & =0.2+0.1 \cos (t) .
\end{array}
$$


Then we have

$$
\begin{array}{lll}
h_{1}=0.2, \quad h_{2}=0.1, & d_{1}=0.2, \quad d_{2}=0.6, \quad \mu_{1}=0.1, \quad \mu_{2}=0.1, \\
& e_{1}=0.1, \quad e_{2}=0.3 .
\end{array}
$$

By using Matlab Toolbox, solving (3.1) we can obtain the feasible solutions

$$
\begin{gathered}
P_{1}=\left[\begin{array}{ll}
0.6720 & 0.0062 \\
0.0062 & 0.7652
\end{array}\right], \quad P_{2}=\left[\begin{array}{ll}
0.7743 & 0.0563 \\
0.0563 & 0.7245
\end{array}\right], \quad Q_{1}=\left[\begin{array}{ll}
1.0529 & 0.0021 \\
0.0021 & 1.2663
\end{array}\right], \\
Q_{2}=\left[\begin{array}{ll}
1.0926 & 0.0036 \\
0.0036 & 1.3185
\end{array}\right], \quad N_{1}=\left[\begin{array}{ll}
0.3602 & 0.0278 \\
0.0278 & 0.3244
\end{array}\right], \quad N_{2}=\left[\begin{array}{ll}
0.3521 & 0.0244 \\
0.0244 & 0.3215
\end{array}\right], \\
N_{3}=\left[\begin{array}{ll}
1.6260 & 0.0284 \\
0.0284 & 1.8337
\end{array}\right], \quad M_{1}=\left[\begin{array}{ll}
0.0070 & 0.0016 \\
0.0016 & 0.0052
\end{array}\right], \quad M_{2}=\left[\begin{array}{cc}
0.0338 & -0.0002 \\
-0.0002 & 0.0353
\end{array}\right], \\
M_{3}=\left[\begin{array}{cc}
0.0891 & -0.0029 \\
-0.0029 & 0.0920
\end{array}\right], \quad R_{1}=\left[\begin{array}{ll}
0.1534 & 0.0253 \\
0.0253 & 0.1688
\end{array}\right], \quad R_{2}=\left[\begin{array}{cc}
0.4415 & -0.0022 \\
-0.0022 & 0.3993
\end{array}\right], \\
R_{3}=\left[\begin{array}{ll}
0.4192 & 0.0053 \\
0.0053 & 0.3845
\end{array}\right], \quad R_{4}=\left[\begin{array}{cc}
1.2767 & -0.0102 \\
-0.0102 & 2.0128
\end{array}\right], \quad U=\left[\begin{array}{ll}
0.0410 & 0.0032 \\
0.0032 & 0.0373
\end{array}\right], \\
V=\left[\begin{array}{ll}
0.1146 & 0.0024 \\
0.0024 & 0.1054
\end{array}\right], \quad \Lambda=\left[\begin{array}{cc}
1.3501 & 0 \\
0 & 2.0371
\end{array}\right] .
\end{gathered}
$$

Hence the Markovian jumping genetic regulatory networks with mode-dependent delays is asymptotically stable. Assume $h_{1}=0.2, \mu_{1}=0.1, \mu_{2}=0.2, e_{1}=0.1, e_{2}=0.5$, and $d_{1}=0.2$. Then we can calculate the maximal allowable bounds of $d_{2}$ with different values of $h_{2}$.

Example 4.2. Consider (3.16) where

$$
\begin{aligned}
& A_{1}=\left[\begin{array}{ll}
5 & 0 \\
0 & 5
\end{array}\right], \quad B_{1}=\left[\begin{array}{ll}
0 & 0 \\
1 & 0
\end{array}\right], \quad C_{1}=\left[\begin{array}{cc}
2.5 & 0 \\
0 & 2.5
\end{array}\right], \quad D_{1}=\left[\begin{array}{ll}
1 & 0 \\
0 & 1
\end{array}\right], \\
& A_{2}=\left[\begin{array}{ll}
5 & 0 \\
0 & 5
\end{array}\right], \quad B_{2}=\left[\begin{array}{ll}
0 & 0 \\
2 & 0
\end{array}\right], \quad C_{2}=\left[\begin{array}{ll}
3 & 0 \\
0 & 3
\end{array}\right], \quad D_{2}=\left[\begin{array}{cc}
0.8 & 0 \\
0 & 0.8
\end{array}\right] \text {. }
\end{aligned}
$$

The uncertain parameters for every mode of the Markovian genetic regulatory networks are given by

$$
\begin{aligned}
& H_{a 1}=\left[\begin{array}{cc}
-0.2 & 0 \\
0 & -0.2
\end{array}\right], \quad H_{b 1}=\left[\begin{array}{cc}
-0.1 & 0.2 \\
0.08 & 0
\end{array}\right], \quad H_{c 1}=\left[\begin{array}{cc}
-0.1 & 0 \\
0 & -0.1
\end{array}\right], \quad H_{d 1}=\left[\begin{array}{cc}
0.15 & 0 \\
0 & 0.15
\end{array}\right] \\
& E_{1}=\left[\begin{array}{cc}
0.3 & 0 \\
0 & 0.3
\end{array}\right], \quad H_{a 2}=\left[\begin{array}{cc}
-0.3 & 0 \\
0 & -0.3
\end{array}\right], \quad H_{b 2}=\left[\begin{array}{cc}
0.1 & 0 \\
0 & 0.1
\end{array}\right], \quad H_{c 2}=\left[\begin{array}{cc}
-0.1 & 0 \\
0 & -0.1
\end{array}\right] \text {, } \\
& H_{d 2}=\left[\begin{array}{cc}
0.1 & 0 \\
0 & 0.1
\end{array}\right], \quad E_{2}=\left[\begin{array}{cc}
0.1 & 0 \\
0 & 0.1
\end{array}\right] \text {. }
\end{aligned}
$$


Table 1: The maximal allowable bounds of $d_{2}$.

\begin{tabular}{lcccc}
\hline Methods & $h_{2}=0$ & $h_{2}=0.5$ & $h_{2}=1$ & $h_{2}=1.5$ \\
\hline Theorem 3.1 & $d_{2}=5.84$ & $d_{2}=2.65$ & $d_{2}=0.46$ & $d_{2}=0.46$ \\
Corollary 3.2 & $d_{2}=5.8$ & $d_{2}=2.635$ & $d_{2}=0.407$ & $d_{2}=0.406$ \\
\hline
\end{tabular}

Table 2: The maximal allowable bounds of $d_{2}$.

\begin{tabular}{lcccc}
\hline Methods & $h_{2}=0.5$ & $h_{2}=1$ & $h_{2}=1.5$ & $h_{2}=2$ \\
\hline Theorem 3.4 & $d_{2}=323$ & $d_{2}=3.475$ & $d_{2}=2.376$ & $d_{2}=2.14$ \\
Corollary 3.5 & $d_{2}=309$ & $d_{2}=3.11$ & $d_{2}=1.97$ & $d_{2}=1.76$ \\
\hline
\end{tabular}

The nonlinear regulation function is taken as $g(x)=x^{2} /\left(1+x^{2}\right)$, so we can easily get $k_{i}=0.65$, the transmission probability is assumed to be $\gamma=\left[\begin{array}{cc}-0.4 & 0.4 \\ 0.6 & -0.6\end{array}\right]$.

Choosing $h_{1}=0.1, h_{2}=0.1, d_{1}=0, d_{2}=0.2, \mu_{1}=0.1, \mu_{2}=0.1, e_{1}=0.2$, and $e_{2}=0.4$. Applying Theorem 3.4 to system (3.16), we can get the following feasible solutions:

$$
\begin{aligned}
& P_{1}=\left[\begin{array}{cc}
3.6297 & -0.0003 \\
-0.0003 & 2.7195
\end{array}\right], \quad P_{2}=\left[\begin{array}{cc}
3.6878 & -0.0003 \\
-0.0003 & 2.5334
\end{array}\right], \quad Q_{1}=\left[\begin{array}{cc}
6.3467 & -0.0029 \\
-0.0029 & 5.0050
\end{array}\right] \\
& Q_{2}=\left[\begin{array}{cc}
5.6235 & -0.0024 \\
-0.0024 & 4.5655
\end{array}\right], \quad N_{1}=\left[\begin{array}{cc}
4.6944 & 0 \\
0 & 3.5184
\end{array}\right], \quad N_{2}=\left[\begin{array}{cc}
4.4643 & 0 \\
0 & 3.2752
\end{array}\right] \text {, } \\
& N_{3}=\left[\begin{array}{cc}
10.6313 & -0.0028 \\
-0.0028 & 7.8495
\end{array}\right], \quad M_{1}=\left[\begin{array}{cc}
0.2268 & 0 \\
0 & 0.2555
\end{array}\right], \quad M_{2}=\left[\begin{array}{cc}
0.2297 & 0 \\
0 & 0.2511
\end{array}\right] \text {, } \\
& M_{3}=\left[\begin{array}{cc}
0.2155 & 0 \\
0 & 0.2095
\end{array}\right], \quad R_{1}=\left[\begin{array}{cc}
0.4985 & 0 \\
0 & 0.4617
\end{array}\right], \quad R_{2}=\left[\begin{array}{ll}
4.0194 & 0.0002 \\
0.0002 & 3.8311
\end{array}\right], \\
& R_{3}=\left[\begin{array}{ll}
3.8486 & 0.0002 \\
0.0002 & 3.6607
\end{array}\right], \quad R_{4}=\left[\begin{array}{cc}
7.0716 & -0.0110 \\
-0.0110 & 3.3522
\end{array}\right], \quad U=\left[\begin{array}{cc}
0.2339 & 0 \\
0 & 0.1500
\end{array}\right] \text {, } \\
& V=\left[\begin{array}{cc}
0.6615 & 0 \\
0 & 0.6188
\end{array}\right], \quad \Lambda=\left[\begin{array}{cc}
5.1026 & 0 \\
0 & 1.6577
\end{array}\right], \quad \varepsilon_{1}=5.4301, \quad \varepsilon_{2}=4.9533 \text {. }
\end{aligned}
$$

Assume $h_{1}=0.1, \mu_{1}=0.1, \mu_{2}=0.1, e_{1}=0.2, e_{2}=0.4$, and $d_{1}=0$. Then we can calculate the maximal allowable bounds of $d_{2}$ with different values of $h_{2}$. results.

It can be seen from Tables 1 and 2 that using the lemma will yield less conservative

\section{Conclusions}

In this paper, we have delt with the robust stability analysis problem for the Markovian jumping genetic regulatory networks with parameter uncertainties and mode-dependent delays. By employing a new Lyapunov-Krasovskii function and a lemma to deal with the terms $-\left(\tau_{i}(t) / d_{2}\right) \xi^{T}(t) \times \epsilon_{1}^{T} R_{1} \epsilon_{1} \xi(t),-\left[1-\left(\tau_{i}(t) / d_{2}\right)\right] \xi^{T}(t) \epsilon_{2}^{T} R_{1} \epsilon_{2} \xi(t),-\left(\sigma_{i}(t) / e_{2}\right) \eta^{T}(t) \epsilon_{4}^{T} R_{3} \epsilon_{4} \eta(t)$, and $-\left[1-\left(\sigma_{i}(t) / e_{2}\right)\right] \eta^{T}(t) \times \epsilon_{5}^{T} R_{3} \epsilon_{5} \eta(t)$, some less conservative sufficient conditions in the terms of LMIs to ensure the robust stability of the addressed Markovian jumping genetic networks 
are derived. Finally, two examples are given to illustrate the usefulness of the derived LMIsbased stability conditions.

\section{Acknowledgments}

This paper was partially supported by the Foundation of Anhui Polytechnic University (2007YQ020) and the National Science Foundation (61203139).

\section{References}

[1] G. Chesi and Y. S. Hung, "Stability analysis of uncertain genetic sum regulatory networks," Automatica, vol. 44, no. 9, pp. 2298-2305, 2008.

[2] B. S. Chen and P. W. Chen, "Robust engineered circuit design principles for stochastic biochemical networks with parameter uncertainties and disturbances," IEEE Transactions on Biomedical Circuits and Systems, vol. 2, no. 2, pp. 114-132, 2008.

[3] C. Li, L. Chen, and K. Aihara, "Synchronization of coupled nonidentical genetic oscillators," Physical Biology, vol. 3, no. 1, pp. 37-44, 2006.

[4] L. Chen and K. Aihara, "A model of periodic oscillation for genetic regulatory systems," IEEE Transactions on Circuits and Systems, vol. 49, no. 10, pp. 1429-1436, 2002.

[5] W. Zhang, Y. Tang, J. Fang, and X. Wu, "Stochastic stability of genetic regulatory networks with a finiteset delay characterization," Chaos, vol. 22, Article ID 023106, 2012.

[6] W. Zhang, J.-a. Fang, and Y. Tang, "Stochastic stability of Markovian jumping genetic regulatory networks with mixed time delays," Applied Mathematics and Computation, vol. 217, no. 17, pp. 72107225, 2011.

[7] W. Zhang, J.-a. Fang, and Y. Tang, "Robust stability for genetic regulatory networks with linear fractional uncertainties," Communications in Nonlinear Science and Numerical Simulation, vol. 17, no. 4, pp. 1753-1765, 2012.

[8] G.-W. Weber, E. Kropat, B. Akteke-Öztürk, and Z.-K. Görgülü, “A survey on OR and mathematical methods applied on gene-environment networks," Central European Journal of Operations Research, vol. 17, no. 3, pp. 315-341, 2009.

[9] Ö. Uğur and G. W. Weber, "Optimization and dynamics of gene-environment networks with intervals," Journal of Industrial and Management Optimization, vol. 3, no. 2, pp. 357-379, 2007.

[10] S. Huang, "Gene expression profiling, genetic networks, and cellular states: an integrating concept for tumorigenesis and drug discovery," Journal of Molecular Medicine, vol. 77, no. 6, pp. 469-480, 1999.

[11] S. A. Kauffman, "Metabolic stability and epigenesis in randomly constructed genetic nets," Journal of Theoretical Biology, vol. 22, no. 3, pp. 437-467, 1969.

[12] S. A. Kauffman, The Origins of Order, Oxford University, New York, NY, USA, 1993.

[13] M. B. Elowitz and S. Leibier, "A synthetic oscillatory network of transcriptional regulators," Nature, vol. 403, no. 6767, pp. 335-338, 2000.

[14] T. Tian, K. Burrage, P. M. Burrage, and M. Carletti, "Stochastic delay differential equations for genetic regulatory networks," Journal of Computational and Applied Mathematics, vol. 205, no. 2, pp. 696-707, 2007.

[15] L. Chen and K. Aihara, "Stability of genetic regulatory networks with time delay," IEEE Transactions on Circuits and Systems, vol. 49, no. 5, pp. 602-608, 2002.

[16] T. Chen, H. He, and G. Church, "Modeling gene expression with differential equations," in Proceedings of the Pacific Symposium on Biocomputing, vol. 4, pp. 29-40, 1999.

[17] A. Becskel and L. Serrano, "Engineering stability in gene networks by autoregulation," Nature, vol. 405, no. 6786, pp. 590-593, 2000.

[18] P. Smolen, D. A. Baxter, and J. H. Byrne, "Mathematical modeling of gene networks," Neuron, vol. 26, no. 3, pp. 567-580, 2000.

[19] Y. Wang, J. Shen, B. Niu, Z. Liu, and L. Chen, "Robustness of interval gene networks with multiple time-varying delays and noise," Neurocomputing, vol. 72, no. 13-15, pp. 3303-3310, 2009.

[20] M. Xiao and J. Cao, "Genetic oscillation deduced from Hopf bifurcation in a genetic regulatory network with delays," Mathematical Biosciences, vol. 215, no. 1, pp. 55-63, 2008.

[21] J. Qiu and J. Cao, "Global synchronization of delay-coupled genetic oscillators," Neurocomputing, vol. 72, no. 16-18, pp. 3845-3850, 2009. 
[22] F. Ren and J. Cao, "Asymptotic and robust stability of genetic regulatory networks with time-varying delays," Neurocomputing, vol. 71, no. 4-6, pp. 834-842, 2008.

[23] J. Cao and F. Ren, "Exponential stability of discrete-time genetic regulatory networks with delays," IEEE Transactions on Neural Networks, vol. 19, no. 3, pp. 520-523, 2008.

[24] Z. Wang, H. Gao, and J. Cao, “On delayed genetic regulatory networks with polytypic uncertainties: robust stability analysis," IEEE Transactions on Nanobioscience, vol. 7, pp. 154-163, 2008.

[25] Y. Sun, G. Feng, and J. Cao, "Stochastic stability of Markovian switching genetic regulatory networks," Physics Letters A, vol. 373, no. 18-19, pp. 1646-1652, 2009.

[26] G. Wang, J. Cao, and J. Liang, "Robust stability of stochastic genetic network with Markovian jumping parameters," Proceedings of the Institution of Mechanical Engineers, vol. 223, no. 6, pp. 797-807, 2009.

[27] L. Huang and X. Mao, "On almost sure stability of hybrid stochastic systems with mode-dependent interval delays," Institute of Electrical and Electronics Engineers, vol. 55, no. 8, pp. 1946-1952, 2010.

[28] Z. Wang, Y. Liu, and X. Liu, "Exponential stabilization of a class of stochastic system with Markovian jump parameters and mode-dependent mixed time-delays," Institute of Electrical and Electronics Engineers, vol. 55, no. 7, pp. 1656-1662, 2010.

[29] A. Ribeiro, R. Zhu, and S. A. Kauffman, "A general modeling strategy for gene regulatory networks with stochastic dynamics," Journal of Computational Biology, vol. 13, no. 9, pp. 1630-1639, 2006.

[30] K. Gu, "An integral inequality in the stability problem of time-delay systems," in Proceedings of the 39th IEEE Confernce on Decision and Control, pp. 2805-2810, Sydney, Australia, December 2000.

[31] S. Boyd, L. El Ghaoui, E. Feron, and V. Balakrishnan, Linear Matrix Inequalities in System and Control Theory, vol. 15 of SIAM Studies in Applied Mathematics, Society for Industrial and Applied Mathematics (SIAM), Philadelphia, Pa, USA, 1994.

[32] Z.-W. Liu and H.-G. Zhang, "Delay-dependent stability for systems with fast-varying neutral-type delays via a PTVD compensation," Acta Automatica Sinica, vol. 36, no. 1, pp. 147-152, 2010. 


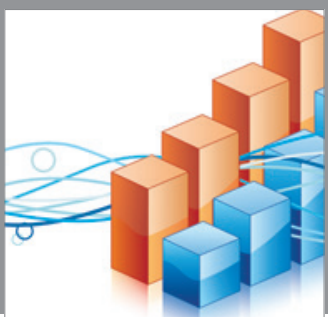

Advances in

Operations Research

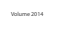

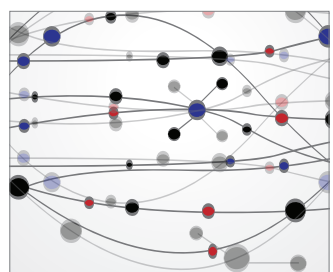

\section{The Scientific} World Journal
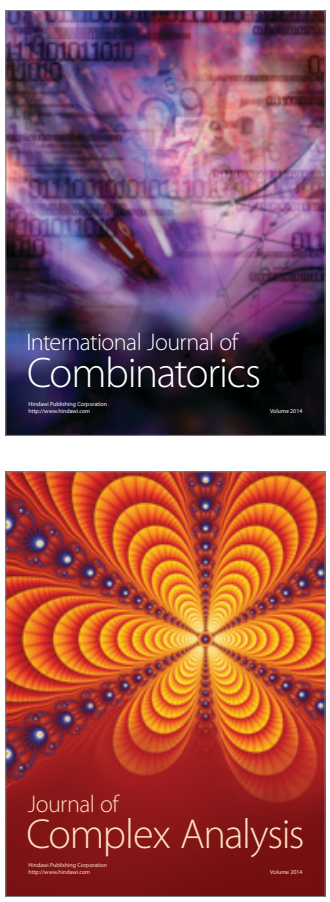

International Journal of

Mathematics and

Mathematical

Sciences
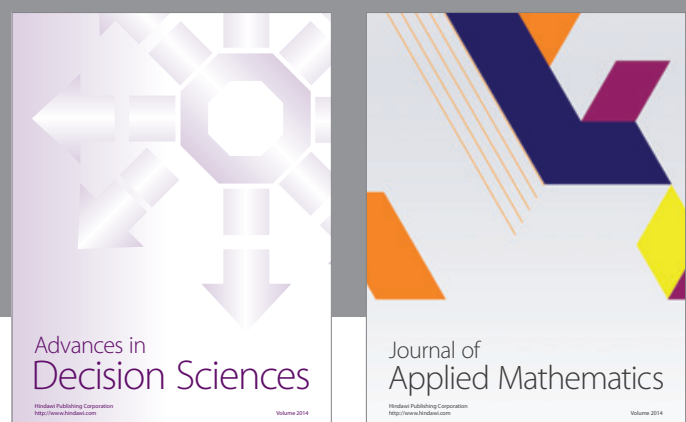

Journal of

Applied Mathematics
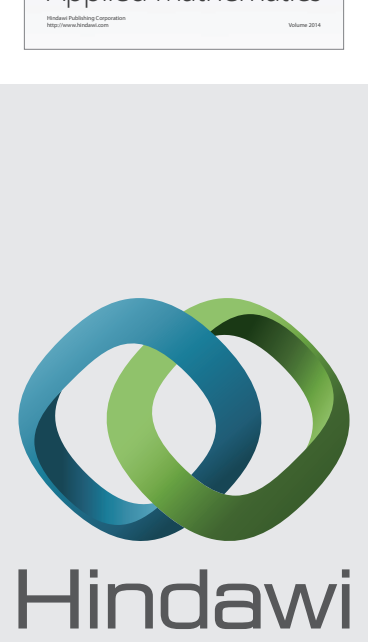

Submit your manuscripts at http://www.hindawi.com
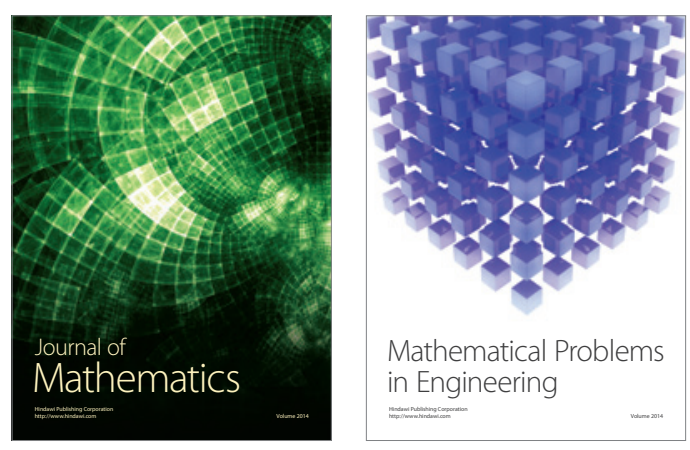

Mathematical Problems in Engineering
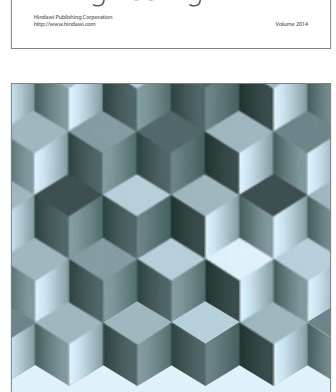

Journal of

Function Spaces
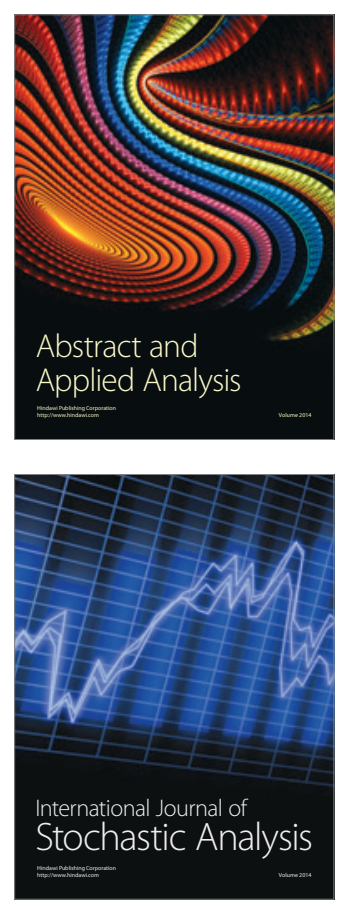

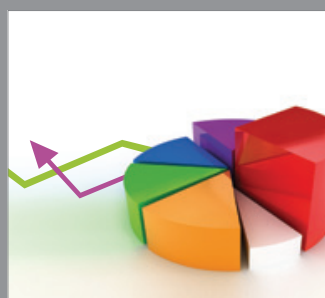

ournal of

Probability and Statistics

Promensencen
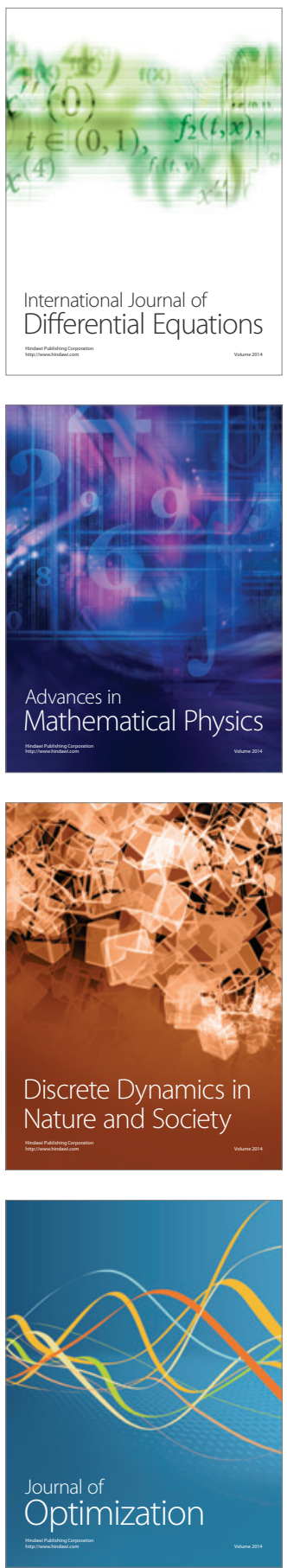